\title{
Seminal plasma antioxidants are directly involved in boar sperm cryotolerance
}

J unwei Li, Isabel Barranco, Asta Tvarijonaviciute, Manuel F. Molina, Emilio A. Martinez, Heriberto Rodriguez-Martinez, Inmaculada Parrilla and J ordi Roca

The self-archived postprint version of this journal article is available at Linköping University Institutional Repository (DiVA):

http:// urn.kb.se/ resolve?urn=urn:nbn:se:liu:diva- 145125

N.B.: When citing this work, cite the original publication.

Li, J., Barranco, I., Tvarijonaviciute, A., Molina, M. F., Martinez, E. A., Rodriguez-Martinez, H., Parrilla, I., Roca, J., (2018), Seminal plasma antioxidants are directly involved in boar sperm cryotolerance, Theriogenology, 107, 27-35. https:/ / doi.org/ 10.1016/j.theriogenology.2017.10.035

Original publication available at:

https:// doi.org/ 10.1016/j.theriogenology.2017.10.035

Copyright: Elsevier

http:// www.elsevier.com/

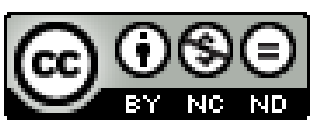


Revised

Seminal plasma antioxidants are directly involved in boar sperm cryotolerance

Junwei $\mathrm{Li}^{1}$, Isabel Barranco ${ }^{1}$, Asta Tvarijonaviciute ${ }^{1}$, Manuel F. Molina ${ }^{1}$, Emilio A. Martinez $^{1}$, Heriberto Rodriguez-Martinez ${ }^{2}$, Inmaculada Parrilla ${ }^{1,3}$, Jordi Roca ${ }^{1,3^{*}}$

${ }^{1}$ Department of Medicine and Animal Surgery, Faculty of Veterinary Science, University of Murcia, Spain; ${ }^{2}$ Department of Clinical and Experimental Medicine (IKE), University of Linköping, Sweden.

${ }^{3}$ Senior authors

*Corresponding author: Jordi Roca (roca@um.es) 


\section{Abstract}

Boar ejaculates are ejected in fractions with a specific composition in terms of sperm numbers and seminal plasma (SP), which is reflected in the varying sperm cryotolerance observed among different fractions. As boar sperm are particularly sensitive to oxidative stress, this study evaluated the role of SP antioxidants in the observed differences in sperm cryotolerance among ejaculate fractions. Ten ejaculates from five boars were manually collected in fractions: the first $10 \mathrm{~mL}$ of the sperm-rich fraction (SRF), the rest of the SRF and the post-SRF. Semen samples comprising the entire ejaculate (EE) were created by proportionally mixing the three fractions described above. Each of the 40 resulting semen samples was split into two aliquots: one was used for sperm cryopreservation following a standard protocol utilizing $0.5-\mathrm{mL}$ straws, and the other was used to collect SP for antioxidant assessment. Frozen-thawed (FT) sperm from the SRF (the first $10 \mathrm{~mL}$ of the SRF and the rest of the SRF) and those from post-SRF were of the highest and worst quality, respectively, which was measured in terms of total and objective progressive motility and viability $(\mathrm{P}<0.01)$. Viable FT sperm from the postSRF generated more reactive oxygen species and experienced more lipid peroxidation than those from the SRF (both the first $10 \mathrm{~mL}$ and the rest of the SRF) $(\mathrm{P}<0.01)$. The percentage of FT sperm exhibiting fragmented nuclear DNA did not differ among ejaculate fractions and the EE. Catalase, glutathione peroxidase and glutathione peroxidase $5(\mathrm{GPx}-5)$ were lowest in SP from the first $10 \mathrm{~mL}$ of the SRF $(\mathrm{P}<0.001)$, whereas superoxide dismutase (SOD) and paraoxonase 1 (PON-1) were highest in SP of the SRF (both the first $10 \mathrm{~mL}$ and the rest of the SRF) $(\mathrm{P}<0.01)$. Trolox-equivalent antioxidant capacity (TEAC) and the ferric-reducing ability of plasma (FRAP) were 
highest in SP from the first $10 \mathrm{~mL}$ of the SRF and lowest in the post-SRF $(\mathrm{P}<0.001)$, whereas cupric-reducing antioxidant capacity was lowest $(\mathrm{P}<0.05)$ in $\mathrm{SP}$ from the first 10 $\mathrm{mL}$ of the SRF. Regression analyses indicated that certain SP antioxidants had good predictive value for post-thaw recovery rates of total motility $\left(\mathrm{R}^{2}=54.8 \%, \mathrm{P}<0.001\right.$; including SOD, TEAC and FRAP) and viability $\left(\mathrm{R}^{2}=56.1 \%, \mathrm{P}<0.001\right.$; including SOD, PON-1, GPX-5 and TEAC). These results demonstrated that certain SP antioxidants are positively involved in boar sperm cryotolerance, minimizing the oxidative stress imposed by cryogenic handling.

Keywords: boar, ejaculate fractions, sperm cryotolerance, seminal plasma, antioxidants.

\section{Introduction}

Existing procedures for the cryopreservation of boar spermatozoa are far from satisfactory [1]. Boar sperm membranes are rich in polyunsaturated fatty acids (PUFA) [2], making them particularly sensitive to oxidative stress (OS) and membrane lipid peroxidation (LPO). The LPO cascade leads to the formation of electrophilic lipid aldehydes, causing a loss of motility and ultimately sperm death [3, 4]. The proportion of PUFA in boar sperm membranes decreases during cryopreservation, likely due to LPO $[5,6]$. An adequate antioxidant system is essential to mitigate OS and avoid LPO. Unfortunately, mammalian sperm lacks such a system $[7,8]$. Therefore, antioxidants present in the seminal plasma (SP) must play a decisive role in protecting sperm against OS [9]. Boar SP contains a wide battery of components with antioxidant properties [10], but their putative role in sperm cryotolerance has not been well characterized. To date, only one study has superficially addressed this topic [11], suggesting that the total 
antioxidant capacity (TAC) of SP is related to boar sperm cryotolerance. The purpose of the present study was to address the role of SP antioxidants in protecting boar sperm against the stress of cryopreservation. Boar ejaculates are emitted in fractions with remarkable differences in terms of both SP composition [12, 13] and sperm cryotolerance $[14,15]$. This peculiarity provides a framework in which a single ejaculate presents different scenarios for sperm-SP interactions, facilitating characterization of the reasons/causes underlying poor boar sperm cryotolerance. In addition to the two most recognized boar ejaculate fractions, the sperm-rich fraction (SRF) and the post-SRF, the first $10 \mathrm{~mL}$ of the SRF are particularly relevant because its sperm exhibits the best cryotolerance, particularly compared to the cryotolerance of the entire ejaculate (EE) [14, 15]. Accordingly, sperm and SP samples from EE and from the three ejaculate fractions described above were used to investigate the relationship between SP antioxidants and boar sperm cryotolerance.

\section{Materials and Methods}

\subsection{Reagents and media}

All chemicals used in this study were analytical grade and purchased from SigmaAldrich Co. (St. Louis, MO, USA). The media were prepared under sterile conditions in a laminar flow hood (MicroH; Telstar, Terrasa, Spain). The basic semen extender was Beltsville Thawing Solution (BTS: $205 \mathrm{mM}$ glucose, $20.39 \mathrm{mM} \mathrm{Na} 3-\mathrm{C}_{6} \mathrm{H}_{5} \mathrm{O}_{7}, 10.0 \mathrm{mM}$ $\mathrm{KCl}, 15.01 \mathrm{mM} \mathrm{NaHCO}$, $3.36 \mathrm{mM}$ EDTA; pH 7.2 and 290-300 mOsmol/kg) supplemented with $50 \mathrm{mg} / \mathrm{mL}$ kanamycin sulfate. EDTA-free phosphate-buffered saline (PBS: $139 \mathrm{mM} \mathrm{NaCl}, 2.7 \mathrm{mM} \mathrm{KCl}, 1.5 \mathrm{mM} \mathrm{KH} 2 \mathrm{PO}_{4}, 8.1 \mathrm{mM} \mathrm{Na} 2 \mathrm{HPO}_{4} \cdot 7 \mathrm{H}_{2} \mathrm{O}$; with 
$0.058 \mathrm{~g} / \mathrm{L}$ penicillin $\mathrm{G}$ and $0.05 \mathrm{~g} / \mathrm{L}$ streptomycin sulfate; $\mathrm{pH}$ 6.8-6.9 and 280-300 mOsmol/kg) was used for flow cytometric analysis. The sperm cooling extender contained 80\% (v:v) Tris-citric acid-glucose extender (111 mM Trizma base, $31.4 \mathrm{mM}$ monohydrate citric acid, 185 mM glucose) and 20\% (v:v) egg yolk, supplemented with $100 \mu \mathrm{g} / \mathrm{mL}$ kanamycin sulfate (pH 7.2 and 295-300 mOsmol/kg). The freezing extender consisted of the FE (89.5\%, v:v) supplemented with glycerol (9\%, v:v) and Equex STM (1.5\%, v:v, Nova Chemical Sales, Scituate, MA, USA) (pH of 6.2 and 1700-1730 $\mathrm{mOsmol} / \mathrm{kg})$.

The fluorescent probes BODIPY 581/591 C11 (BODIPY), 5-(and-6) chloromethyl-20,70-dichlorodihydrofluorescein diacetate acetyl ester (CM- ${ }_{2}$ DCFDA), dihydroethidium (DHE), MitoSOX ${ }^{\mathrm{TM}}$ Red (MSR), SYTOX ${ }^{\mathrm{TM}}$ Green (SYTOX) and propidium iodide (PI) were purchased from Invitrogen ${ }^{\mathrm{TM}}$ (Thermo Fisher Scientific, Waltham, MA, USA). The fluorescent probes Hoechst 33342 (H42) and fluorescein isothiocyanate-conjugated peanut agglutinin (FITC-PNA) were purchased from Sigma Aldrich.

\subsection{Semen and SP samples}

The semen and SP samples used in the experiment were collected from five mature (2 to 3 years old) and healthy fertile boars (Landrace and Large White) housed in a commercial artificial insemination (AI) center (AIM Iberica, Calasparra, Murcia, Spain) and regularly used in AI programs (two ejaculates collected per week). Boars were housed under environmentally controlled conditions (temperature $15-25^{\circ} \mathrm{C}$, natural light from windows) and were given commercial feed, according to semen donor requirements. Ejaculates (two per boar) were collected in fractions using the gloved-hand method. 
Specifically, the first $10 \mathrm{~mL}$ of the SRF, the rest of the SRF and the post-SRF. EE samples were created by proportionally mixing the three described ejaculate fractions. A total of 40 semen samples were collected to perform the study. Each of the 40 semen samples was split into two aliquots immediately after collection. One aliquot was extended 1:1 (v:v) with BTS and stored in an insulated container at $15-17^{\circ} \mathrm{C}$, while the other aliquot was immediately centrifuged twice at $1,500 \mathrm{x}$ g for $10 \mathrm{~min}$ to separate the sperm from the SP. The resultant SP samples were microscopically examined to confirm that they were sperm free and were then stored in cryotubes and frozen at $-20^{\circ} \mathrm{C}$. Semen and SP samples were transported to the Andrology Laboratory of the Veterinary Teaching Hospital of the University of Murcia, where they arrived in less than $1 \mathrm{~h}$. At the laboratory, semen samples remained stored at $17{ }^{\circ} \mathrm{C}$ (cooled incubator FOC 120E, VELP Scientifica, Usmate, Italy) overnight and were frozen the next day. The SP samples remained stored at $-80{ }^{\circ} \mathrm{C}$ (Ultra-Low Freezer, Haier, Canada) until antioxidant analyses were performed.

\subsection{Measurement of SP antioxidant capacity}

SP TAC was measured in terms of the Trolox equivalent antioxidant capacity (TEAC), ferric-reducing ability of plasma (FRAP) and cupric-reducing antioxidant capacity (CUPRAC), all of which measure the activity of non-enzymatic antioxidants using different approaches. The TEAC method is based on a color change by 2,2'azinobis-3-ethylbenzothiazoline-6-sulfonate [16], the FRAP method is based on the reduction of $\mathrm{Fe}^{3+}$ to $\mathrm{Fe}^{2+}[17]$ and the CUPRAC method is based on the reduction of $\mathrm{Cu}^{2+}$ to $\mathrm{Cu}^{1+}[18]$. These assays were validated to SP samples in preliminary assays.

The enzymatic antioxidant activity of SP was measured as the activity of catalase (CAT), total glutathione peroxidase (GPOx), superoxide dismutase (SOD) and 
paraoxonase 1 (PON-1), and the concentration of glutathione peroxidase 5 (GPx-5). CAT, GPOx and SOD activity levels were measured using commercially available assays following the manufacturers' instructions (CAT: Sigma-Aldrich, St. Louis, MO, USA; GPOx and SOD: Randox, Crumlin, UK). PON-1 activity was assessed by measuring the hydrolysis of p-nitrophenyl acetate to p-nitrophenol, as described by Barranco et al. (2015) for SP [19]. The concentration of GPx-5 was measured using a commercially available sandwich enzyme-linked immunosorbent assay following the manufacturer's instructions (Mybiosource, San Diego, California, USA). All measurements, with the exception of the GPx-5 concentration and CAT activity, were performed using an automated biochemistry analyzer (Olympus AU600 Automatic Chemistry Analyzer, Olympus Europe GmbH, Germany) with an inter- and intra-assay imprecision of less than 15\%. The GPx-5 concentration and CAT activity were measured using a micro-plate reader (PowerWave XS; Bio-Tek Instruments). The results of the TAC assays (TEAC, FRAP and CUPRAC) were expressed in mmol/L. SOD, CAT and PON-1 activities were expressed as IU/mL, and GPOx activity was expressed as IU/L. The concentration of GPx-5 was expressed as $\mathrm{ng} / \mathrm{mL}$.

\subsection{Sperm cryopreservation}

The semen samples were frozen using a $0.5-\mathrm{mL}$ straw freezing procedure described by Alkmin et al. (2014) [15]. Briefly, the samples were centrifuged (3 min at 2,400 × g; Megafuge 1.0 R, Heraeus, Hanau, Germany), and sperm pellets were resuspended in cooling extender to a concentration of $1.5 \times 10^{9} \mathrm{sperm} / \mathrm{mL}$. After cooling to $5{ }^{\circ} \mathrm{C}$ for $150 \mathrm{~min}$, extended sperm samples were re-extended in the freezing extender to a final concentration of $1.0 \times 10^{9}$ sperm/mL. Immediately, the extended sperm samples 
were packed into 0.5-mL polyvinyl chloride (PVC) French straws (Minitüb, Tiefenbach, Germany) and frozen in liquid nitrogen ( $\mathrm{LN}_{2}$ ) vapors (placed on a metal rack, $3 \mathrm{~cm}$ above liquid nitrogen surface for $20 \mathrm{~min}$ ). The frozen straws were stored in a $\mathrm{LN}_{2}$ container for at least one week. Straws were then thawed in a circulating water bath at $37^{\circ} \mathrm{C}$ for $20 \mathrm{~s}$ and extended with BTS (1:1, v:v). The resulting extended FT sperm samples remained at $37^{\circ} \mathrm{C}$ in the dark for $150 \mathrm{~min}$, and sperm quality and functionality were evaluated at 30 and 150 min after thawing.

\subsection{Sperm assessments}

Sperm were evaluated in terms of their total and progressive motility, viability, nuclear DNA fragmentation, intracellular ROS generation and LPO.

Sperm motility was objectively evaluated using an integrated sperm analysis system (ISASV1 ${ }^{\circledR}$; Proiser R+D, Paterna, Spain). Briefly, sperm samples were extended with BTS to a final concentration of $20-30 \times 10^{6}$ sperm/mL. For each evaluation, $5 \mu \mathrm{L}$ of sample was placed in a Makler counting chamber (Sefi Medical Instruments, Haifa, Israel) pre-warmed to $38{ }^{\circ} \mathrm{C}$. Then, four or five fields were captured to analyze a minimum of 400 sperm per sample. The following sperm motility variables were recorded: percentages of total motile spermatozoa (with an average path velocity $\geq 20$ $\mu \mathrm{m} / \mathrm{s}$ ) and of sperm exhibiting rapid and progressive movement (straight line velocity $\geq$ $40 \mu \mathrm{m} / \mathrm{s})$.

Sperm viability was cytometrically evaluated (BD FACSCanto II cytometer; Becton Dickinson Co, Franklin Lakes, NJ, USA) in terms of plasma and acrosome membrane integrity. One hundred microliters of each sperm sample $\left(30 \times 10^{6} \mathrm{sperm} / \mathrm{mL}\right.$ in BTS) was stained with $3 \mu \mathrm{L}$ of $\mathrm{H}-42(0.05 \mathrm{mg} / \mathrm{mL}$ in PBS), $2 \mu \mathrm{L}$ of PI $(0.5 \mathrm{mg} / \mathrm{mL}$ in 
PBS) and $2 \mu \mathrm{L}$ of PNA-FITC (200 $\mu \mathrm{g} / \mathrm{mL}$ in PBS) and incubated in the dark at $37^{\circ} \mathrm{C}$ for 10 min. Four hundred microliters of PBS were added to each sample prior to cytometry analysis. A total of 10,000 H-42 positive events were recorded for each sample, and both the PI and PNA-FITC negative sperm population were viable.

Total and progressive sperm motility and sperm viability were measured after thawing and were recorded as both raw and recovery data. Raw data were recorded for thawed sperm samples, whereas recovery data consists of the percentages of motile and viable sperm relative to the percentages before freezing, calculated using the following equation: (raw post-thaw sperm quality $\times 100$ )/ sperm quality before freezing).

Intracellular ROS generation was evaluated by measuring superoxide ion $\left(\mathrm{O}_{2}{ }^{{ }^{-}}\right)$ and hydrogen peroxide $\left(\mathrm{H}_{2} \mathrm{O}_{2}\right)$ levels. Mitochondrial $\mathrm{O}_{2}{ }^{\cdot-}$ generation was assessed using MSR following a procedure described by Koppers et al. (2008) [20] with slight modifications. Briefly, $1 \mathrm{~mL}$ of each sperm sample $\left(20 \times 10^{6}\right.$ sperm $\left./ \mathrm{mL}\right)$ was mixed with $0.5 \mu \mathrm{L}$ of MSR (5 mM in DMSO) and incubated at $37^{\circ} \mathrm{C}$ in the dark for $15 \mathrm{~min}$. Thereafter, the samples were centrifuged $(5 \mathrm{~min}$ at $600 \times \mathrm{g}$ ) and sperm pellets were resuspended in PBS to $10 \times 10^{6}$ sperm/mL. Then, sperm samples were mixed with $10 \mu \mathrm{L}$ of SYTOX (5 $\mu \mathrm{M}$ in DMSO) and $15 \mu \mathrm{L}$ of $\mathrm{H}-42$ (0.05 mg/mL in PBS). Similar sperm samples, mixed with $50 \mu \mathrm{L}$ of Rotenone (0.01 mM in DMSO), were used as the positive control. The sperm samples were incubated in the dark at $37^{\circ} \mathrm{C}$ for 15 min immediately prior to cytometric analysis and extended in PBS to $3 \times 10^{6} \mathrm{sperm} / \mathrm{mL}$. Sperm positive for H-42 and MSR and negative for SYTOX were considered viable, with high mitochondrial $\mathrm{O}_{2}{ }^{\bullet-}$ generation.

The total generation of $\mathrm{O}_{2}{ }^{--}$was assessed using DHE following a procedure described by Koppers et al. (2008) [20] with slight modifications. Briefly, $1 \mathrm{~mL}$ of each sperm sample $\left(10 \times 10^{6}\right.$ sperm/mL $)$ was mixed with $7.5 \mu \mathrm{L}$ of $\mathrm{H}-42(0.05 \mathrm{mg} / \mathrm{mL}$ in PBS $)$, 
$10 \mu \mathrm{L}$ of SYTOX (5 $\mu \mathrm{M}$ in DMSO) and $10 \mu \mathrm{L}$ of DHE (200 $\mu \mathrm{M}$ in DMSO) and incubated in the dark at $37^{\circ} \mathrm{C}$ for $15 \mathrm{~min}$. Thereafter, the sperm samples were centrifuged ( $5 \mathrm{~min}$ at $600 \times \mathrm{g}$ ), and the sperm pellets were re-suspended in PBS to $3 \times 10^{6} \mathrm{sperm} / \mathrm{mL}$. The sperm population that was H-42-positive, SYTOX Green-negative and DHE-positive was considered viable, with high total intracellular $\mathrm{O}_{2}{ }^{\circ-}$ generation.

Intracellular $\mathrm{H}_{2} \mathrm{O}_{2}$ generation in FT viable sperm was measured using CM$\mathrm{H}_{2}$ DCFDA following a procedure described by Guthrie and Welch (2006) [21]. Briefly, $50 \mu \mathrm{L}$ of each sperm sample $\left(30 \times 10^{6} \mathrm{sperm} / \mathrm{mL}\right)$ was extended in $950 \mu \mathrm{L}$ of PBS containing $1.5 \mu \mathrm{L}$ of $\mathrm{H}-42(0.05 \mathrm{mg} / \mathrm{mL}$ in PBS $), 1 \mu \mathrm{L}$ of PI $(0.5 \mathrm{mg} / \mathrm{mL}$ in PBS) and 1 $\mu \mathrm{L}$ of CM-H $\mathrm{H}_{2}$ DCFDA (1 mM in DMSO). A similar sperm sample, mixed with $1 \mu \mathrm{L}$ of tert-butyl hydroperoxide (TBH) solution (70\% in distilled water), was used as a positive control. The sperm samples were incubated in the dark at $37{ }^{\circ} \mathrm{C}$ for 30 min prior to cytometric analysis. Sperm negative for PI and positive for DCF were considered viable, with high intracellular $\mathrm{H}_{2} \mathrm{O}_{2}$ generation.

Sperm membrane LPO was evaluated using BODIPY following a procedure described by Koppers et al. (2008) [20] with slight modifications. Briefly, sperm samples $\left(20 \times 10^{6}\right.$ sperm/mL in PBS) were mixed with $2.5 \mu \mathrm{L}$ of BODIPY and incubated in the dark at $37^{\circ} \mathrm{C}$ for $30 \mathrm{~min}$. Thereafter, sperm samples were centrifuged $(7 \mathrm{~min}$ at $300 \times \mathrm{g}$ ) and sperm pellets were re-suspended in $1 \mathrm{~mL}$ of PBS. Then, $100 \mu \mathrm{L}$ of each sperm sample was mixed with $10 \mu \mathrm{L}$ of PI (0.5 mg/mL in PBS) and $2 \mu \mathrm{L}$ of $\mathrm{H}-42(0.05 \mathrm{mg} / \mathrm{mL}$ in PBS) and incubated in the dark at $37^{\circ} \mathrm{C}$ for $10 \mathrm{~min}$. A similar sperm sample, mixed with $9 \mu \mathrm{L}$ of TBH (70\% in distilled water) and incubated at $37{ }^{\circ} \mathrm{C}$ in the dark for $30 \mathrm{~min}$, was used as a positive control. Four hundred microliters of PBS were added to each sperm sample prior to cytometric analysis. The H-42-positive, PI-negative and BODIPY-positive sperm population was considered viable exhibiting LPO. 
Nuclear DNA fragmentation in FT sperm was evaluated immediately after thawing using the Sperm-Sus-Halomax ${ }^{\circledR}$ kit (Halotech DNA SL, Madrid, Spain) following a procedure described by Alkmin et al. (2013) [22]. A minimum of 300 sperm were microscopically evaluated for each FT sperm sample $\left(10 \times 10^{6} \mathrm{sperm} / \mathrm{mL}\right.$ in EDTAfree BTS), and sperm exhibiting a large scattered halo around the head were considered to have fragmented nuclear DNA.

\subsection{Statistical analysis}

Statistical analyses were performed using the IBM SPSS statistics 19.0 package (IBM Spain, Madrid) and following the recommendations of Petrie and Watson (2013) [23]. The normality of the data set was confirmed based on the residuals using the Kolmogorov-Smirnov test. A repeated-measures ANOVA was performed to evaluate the influence of the semen source (first $10 \mathrm{~mL}$ of the SRF, rest of the SRF, post-SRF and EE) on the sperm quality (raw and recovery rates), the intracellular ROS-generation and the LPO of FT sperm at two post-thaw evaluation times (30 and 150 min post-thaw). Mixed ANOVA tests, using boar and trial as random effects, were performed to evaluate the influence of semen source on both nuclear DNA fragmentation in FT sperm and antioxidant measurements in SP. Multivariable linear regression analysis, performed with the forward stepwise method to choose explanatory variables, was used to identify SP antioxidants related to variability in the post-thaw recovery rates of total sperm, progressive motility and sperm viability. The Bonferroni test was used for post hoc analyses where appropriate. A value of $\mathrm{P}<0.05$ was accepted as the minimum level of significance. 


\section{Results}

Sperm quality was measured in terms of both total and progressive motility as well as viability, and the percentages found for them in fresh semen samples were, respectively, $80.30 \pm 1.76,28.20 \pm 2.16$ and $84.28 \pm 1.55$ in the $10 \mathrm{~mL}$ of SRF; $80.90 \pm$ 2.39, $25.70 \pm 3.20$ and $81.06 \pm 2.43$ in the rest of SRF; $69.60 \pm 4.25,27.10 \pm 2.73$ and $71.40 \pm 3.63$ in the post-SRF; and $75.50 \pm 2.71,23.70 \pm 2.52$ and $76.14 \pm 3.34$ in the entire ejaculate.

After thawing, sperm quality was measured at 30 and 150 min and recorded as raw and recovery rates. The two major factors, semen source and post-thaw evaluation time, influenced $(\mathrm{P}<0.01)$ all sperm quality parameters evaluated. Sperm quality at 150 min was worse $(\mathrm{P}<0.05)$ than that at 30 min post-thaw. The interaction between sperm source and post-thaw evaluation time was not significant for any of the sperm quality parameters assessed, regardless of whether they were recorded as raw or recovery rates. Accordingly, Table 1 shows the averaged data of the two post-thaw evaluation times for each semen source. The FT sperm of the SRF (the first $10 \mathrm{~mL}$ of the SRF and the rest of the SRF) exhibited the best post-thaw quality, whereas FT sperm from post-SRF exhibited the worst post-thaw quality, irrespective of whether the data were recorded as raw or recovery rates. The post-thaw quality of FT sperm from EE was also worse than that of FT sperm from the SRF (the first $10 \mathrm{~mL}$ of the SRF and the rest of the SRF) but was better than that from the post-SRF.

Intracellular ROS generation was measured in FT viable sperm in terms of the capacity to generate $\mathrm{O}_{2}{ }^{-}$, , both in the entire cell (primarily in the cytoplasm and membrane) and specifically in the mitochondrial sheath, and $\mathrm{H}_{2} \mathrm{O}_{2}$. Semen source and post-thaw incubation time influenced $(\mathrm{P}<0.05)$ ROS generation, but the interaction 
between these factors was not significant. Therefore, the measurements recorded at both post-thaw evaluation times were averaged for each semen source (Figure 1). The generation of $\mathrm{O}_{2}{ }^{\cdot-}$ showed a similar pattern in both the mitochondria (Figure $1 \mathrm{~A}$ ) and the cytoplasm and membrane (Figure 1B), characterized by lower levels in the SRF (both in first $10 \mathrm{~mL}$ and the rest of the SRF) and higher levels in the post-SRF. $\mathrm{H}_{2} \mathrm{O}_{2}$ generation in FT viable sperm also differed among semen sources $(\mathrm{P}<0.01)$, with those from the post-SRF having the highest percentages (Figure 1C). The percentage of FT viable sperm showing LPO also differed $(\mathrm{P}<0.01)$ among semen sources, with those from the SRF (the first $10 \mathrm{~mL}$ of the SRF and the rest of the SRF) and the post-SRF having the lowest and highest percentages, respectively (Figure 1D).

Sperm nuclear DNA status was evaluated once in each semen sample immediately after thawing. The percentage of FT sperm showing fragmented nuclear DNA was always below 4\%, without significant differences among semen sources (Figure 2).

The total antioxidant capacity of SP was measured in terms of TEAC, FRAP and CUPRAC (Figure 3). TEAC (Figure 3A) and FRAP (Figure 3B) measurements were highest in the SP from the first $10 \mathrm{~mL}$ of the SRF and lowest in the post-SRF $(\mathrm{P}<0.001)$. In contrast, CUPRAC measurements were lowest $(\mathrm{P}<0.05)$ in the SP from the first $10 \mathrm{~mL}$ of the SRF compared to other ejaculate fractions and the recomposed EE (Figure 3C).

Five SP enzymes with antioxidant capacity were evaluated, specifically CAT, GPOx, GPx-5, SOD and PON-1 (Figure 4). CAT and GPOx activities and the concentration of GPx-5 showed similar patterns (Figures 4A-C). They were lower in the SP from the first $10 \mathrm{~mL}$ of the SRF than in the other ejaculate fractions and the recomposed EE $(\mathrm{P}<0.05)$. The SP activities of SOD (Figure 4D) and PON-1 (Figure 4E) also showed a similar pattern, with higher levels in the SP of the SRF (both the first 10 $\mathrm{mL}$ and the rest of the SRF) than in the post-SRF and the recomposed $\mathrm{EE}(\mathrm{P}<0.001)$. 
Using the forward stepwise method to choose explanatory variables, multiple linear regression analyses generated 3, 2 and 4 different regression models to predict the recovery rates of total motility, progressive motility and sperm viability after thawing, respectively, for each of the two post-thaw evaluation times. The models generated for each dependent or response variable were similar for the two post-thaw evaluation times. For this reason, the final models were generated based on the combination of data recompiled at the two post-thaw evaluation times. To simplify the presentation of the results, only the regression model with the highest value for adjusted $\mathrm{R}^{2}$ was considered for each of the response variables. The model chosen for the post-thaw recovery rates of total sperm motility had a predictive value of $54.8 \%(F=35.32 ; \mathrm{P}<0.001)$ and included SOD, FRAP and TEAC as explanatory variables. The model for post-thaw recovery rates of progressive motility had a predictive value of $27.1 \%(F=16.77 ; \mathrm{P}<0.001)$ and included SOD and PON-1 as explanatory variables. Finally, the model for post-thaw recovery rates of sperm viability had a predictive value of $56.1 \%(F=28.20$; $P<0.001)$, including SOD, PON-1, TEAC and GPX-5 as explanatory variables. In summary, the regression models generated to explain the variability in the post-thaw recovery rates of total motility and viability showed good predictive value because they explained more than half of the total variation. In contrast, the model chosen to explain the variability in post-thaw recovery rates of progressive motility had a weak predictive value because it explained less than $30 \%$ of the total variation.

\section{Discussion}

The results of the present study show for the first time a clear relationship between SP components with antioxidant capacity and the capability of boar spermatozoa to 
sustain cryopreservation. Here, SOD, PON1, GPx-5 and two TAC measurements, specifically TAEC and FRAP, were the SP antioxidants demonstrating the clearest roles.

The above conclusion was reached after first observing differences in sperm cryotolerance among different ejaculate fractions. This is a well-known issue in that the spermatozoa from the first $10 \mathrm{ml}$ of the SRF are known to freeze better than those from the entire ejaculate [15, 24, 25]. The results of the present study, in addition to agreeing with these findings, demonstrated the poor cryotolerance of spermatozoa from the postSRF, as only 20 and 35\% of the motile and viable sperm before freezing were recovered after thawing. The post-SRF contributes approximately 2/3 of the total SP of EE as well as approximately 10 to $15 \%$ of spermatozoa [26]. Thus, the extreme cryosensitivity of the post-SRF sperm is the primary cause of the poor cryotolerance of EE sperm compared with that of the SRF (the first $10 \mathrm{~mL}$ and the rest of the SRF). Noticeable that AI centers are currently moving away from collecting only the SRF towards collecting the entire EE [27], which, as demonstrated here, negatively impacts sperm cryotolerance. This must be considered before creating boar sperm cryobanks.

The peculiar membrane lipid architecture, specifically the high proportion of PUFA, of boar spermatozoa makes them particularly sensitive to OS [5, 6]. However, there is currently controversy regarding whether the cryopreservation process itself causes OS in boar spermatozoa (reviewed by Yeste, 2016 [1]). The widespread successful use of antioxidants as additives for freezing and/or thawing extenders to improve the quality and functionality of boar FT sperm (reviewed by Bathgate, 2011 [9]) indirectly supports the prediction that boar sperm undergo OS during cryopreservation. Boar sperm are believed to acquire OS sensitivity immediately before freezing, specifically during the cooling period, and the consequences are particularly evident after thawing $[28,29]$. The present study evaluated the OS experienced by viable FT sperm in terms of 
intracellular ROS generation and LPO. Differences among the ejaculate fractions in terms of the levels of ROS generated by viable FT sperm were clear: samples from the postSRF generated more ROS than those from the SRF (both the first $10 \mathrm{~mL}$ and the rest of the SRF). Viable FT sperm from the post-SRF also experienced more LPO than those from the SRF. Despite the lack of a differential threshold between beneficial or deleterious levels of ROS generated by spermatozoa [30], increased levels of ROS are known to lead to OS and thus cause sperm dysfunction [31]. Therefore, based on our results, the cryopreservation process causes OS in boar spermatozoa, but the intensity of OS varies substantially between ejaculate fractions. This is particularly evident in FT sperm from the post-SRF. The intensity of OS is likely related to post-thaw sperm quality because viable FT sperm from the post-SRF, which experience greater OS, exhibited the worst post-thaw sperm quality. In contrast, FT sperm from the SRF (both the first $10 \mathrm{~mL}$ of the SRF and the rest of the SRF) followed an opposite trend, showing less OS and better post-thaw sperm quality. Unsurprisingly, there was a low incidence of FT sperm with fragmented nuclear DNA in the three ejaculate fractions and EE, and there were no differences among them. Nuclear DNA fragmentation is the ultimate sperm target of OS attack, which is particularly evident in the FT sperm of many mammalian species [32]. The nuclear DNA of boar sperm withstands the cryopreservation process [22, 33, 34] because it only contains protamine 1 for DNA packaging. This provides high stability under stressful conditions such as cryopreservation or OS [35].

Currently, the reasons underlying the differences in sperm cryotolerance among ejaculate fractions remain unclear. However, the source of these differences is more likely found in the SP rather than in the sperm themselves. Saravia et al. [14] and Alkmin et al. [15] proved that SP influences freezability of boar sperm and that it does this differently depending on the fraction it came from. The SP source differs among different ejaculate 
fractions. For the first $10 \mathrm{~mL}$ of the SRF, the epididymis and prostate supply the SP. For the remainder of the SRF, the prostate and, to a lesser extent, the vesicular glands supply the SP. Finally, for the post-SRF, the vesicular glands and, to a lesser extent, the bulbourethral glands supply the SP [26]. Accordingly, differences in SP composition, generally described in more qualitative than quantitative terms, have been well documented among ejaculate fractions [12, 13, 36]. This may explain the recorded differences in sperm cryotolerance. At present, however, little is known about the SP components implicated. There have been few studies addressing this subject, and they focused on searching for SP proteins linked to boar sperm cryotolerance [37-39].

Porcine spermatozoa are particularly sensitive to OS, and cryopreservation increases the incidence of LPO [6]. The present study demonstrates clear differences between ejaculate fractions in terms of the intensity of OS experienced by FT sperm, as described above. Consequently, the study focused on SP antioxidants to explain differences between the ejaculate fractions in terms of sperm cryotolerance. Currently, there are no procedures available to assess the TAC of an organic fluid such as SP [40]. Accordingly, the present study evaluated antioxidants, either individually or grouped. SP enzymes with proven antioxidant capacity, specifically SOD, CAT, GPOx, GPx-5 and PON-1, were individually evaluated. SP non-enzymatic antioxidants, such as ascorbic acid, $\alpha$-tocopherol and glutathione, were evaluated together using non-specific TAC tests: TEAC, CUPRAC and FRAP. There were substantial quantitative differences among the ejaculate fractions for several of the SP antioxidants measured. Interestingly, the first 10 $\mathrm{mL}$ of the SRF and the post-SRF were the ejaculate fractions containing SP with the best and worst antioxidant capacity, respectively. The same fractions showed the best and worst ROS generation, LPO and sperm cryotolerance, respectively. Differences in antioxidant capacity between these two ejaculated fractions were particularly evident for 
SOD and TAC measurements (TEAC, CUPRAC and FRAP). Indeed, TAEC is the only SP antioxidant measurement suggested thus far as a potential marker of boar sperm cryotolerance [11].

Regression models were used to identify SP antioxidants that explain differences in sperm cryotolerance and to determine whether these differences are powerful enough to explain the observed variability in the post-thaw recovery rates of total and progressive motility and viability. The models showed good predictive value for total motility and viability and they included SOD, TEAC and FRAP as explanatory variables for total motility, whereas that for viability included SOD, PON-1, GPX-5 and TEAC. SOD was positively related to the post-thaw recovery rates of total motility and viability. These results support previous studies highlighting the relevance of SOD activity for successful sperm cryotolerance [41, 42]. SOD is an essential antioxidant for avoiding OS because it initiates a cascade of reactions intended to neutralize the more harmful ROS [43]. Specifically, SOD neutralizes $\mathrm{O}_{2}{ }^{\circ}$, the primary ROS generated by spermatozoa in both the mitochondrial and plasma membranes, which, in excess, triggers a dangerous oxidative chain reaction leading to LPO and thereby to the loss of motility and sperm death [44]. Regression analysis identified two other antioxidant enzymes that were positively related to boar sperm cryotolerance: PON-1 and GPx-5. PON-1 binds to membrane cholesterol, preventing its oxidation and thereby positively influencing both motility attributes and the sperm membrane integrity [45]. GPx-5, one of the eight enzymes included in the GPx family, is the most abundant in the male genital tract and, together with glutathione, catalyzes reactions to inactivate hydroperoxides [46].

Two tests measuring SP-TAC were also identified in the regression analysis as positively related to sperm cryotolerance: TEAC and FRAP. Both TAC assays measure the antioxidant capacity of ascorbic acid, uric acid, $\alpha$-tocopherol and glutathione [17, 47]. 
Ascorbic acid and $\alpha$-tocopherol are inserted into the membrane structure and are particularly efficient at reducing ROS that is superficially attached to the membranes. Specifically, ascorbic acid reduces nitroxide radicals, and $\alpha$-tocopherol reduces lipid peroxyl radicals. Moreover, both are individually sufficient to minimize membrane LPO when present at appropriate concentrations [48]. Uric acid is another powerful ROS scavenger that binds to the plasma membrane but is not effective at minimizing LPO [48]. Reduced glutathione is likely the most important non-enzymatic SP-ROS scavenger, and it acts primarily by neutralizing hydroxyl radicals. Hydroxyl radicals are among the most dangerous ROS generated from inactivated $\mathrm{H}_{2} \mathrm{O}_{2}$ [9]. FRAP more specifically measures a set of antioxidants capable of reducing ferric $\mathrm{Fe}^{+3}$ to $\mathrm{Fe}^{+2}$, making them effective for neutralizing hydroperoxides [17]. These results are supported by empirical studies demonstrating the ability of SOD, citric acid, $\alpha$-tocopherol and glutathione for improving post-thaw sperm quality when they are used as additives of freezing extenders [41, 49, 50]. Of note, the ability of Trolox, a vitamin E analogue, to improve boar sperm cryopreservation differs among ejaculate fractions [24].

A final question remains: when the above-mentioned SP antioxidants protect boar sperm against cryopreservation stress? Clarifying this issue is particularly relevant in pigs because SP does not participate in the freezing and thawing of sperm, as it is removed prior to freezing to facilitate suitable extension of the sperm with the freezing extender [1]. Thus, only two scenarios are possible. Either SP antioxidants act during ejaculation and/or they act during the holding time prior to freezing. The holding time is typically between 16 and $20 \mathrm{~h}$, during which the sperm remain surrounded by their own SP, facilitating interactions between the spermatozoa and SP components. This holding time results in sufficient recovery rates of functional spermatozoa after thawing [51]. In either of the two scenarios, SP antioxidants work against OS prior to freezing, making the sperm 
more resilient to the stress of cryopreservation. In contrast, sperm are sensitive to cryopreservation stress when the potential activity of the above-mentioned SP antioxidants is reduced. Thus, OS increased after thawing in FT sperm from post-SRF.

In summary, this study demonstrated that certain SP antioxidants are directly involved in the cryotolerance of boar spermatozoa. Boar FT sperm experience OS but demonstrate different intensity levels in different ejaculate fractions. These differences are likely attributable to variations in the concentration and/or activity of SP antioxidants among the ejaculate fractions.

\section{Conflict of interest}

The authors have declared no conflict of interest.

\section{Acknowledgements}

The authors are grateful to AIM Iberica (Topigs Norsvin España) for providing the semen samples. The study was supported by the Seneca Foundation Murcia of Spain

(19892/GERM-15). Junwei Li was financially supported by the China Scholarship Council.

\section{References}

[1] Yeste M. Sperm cryopreservation update: Cryodamage, markers, and factors affecting the sperm freezability in pigs. Theriogenology. 2016;85:47-64. 
[2] Flesch FM, Brouwers JF, Nievelstein PF, Verkleij AJ, van Golde LM, Colenbrander B, et al. Bicarbonate stimulated phospholipid scrambling induces cholesterol redistribution and enables cholesterol depletion in the sperm plasma membrane. $\mathrm{J}$ Cell Sci. 2001;114:3543-55.

[3] Awda BJ, Mackenzie-Bell M, Buhr MM. Reactive oxygen species and boar sperm function. Biol Reprod. 2009;81:553-61.

[4] Aitken RJ, Baker MA, Nixon B. Are sperm capacitation and apoptosis the opposite ends of a continuum driven by oxidative stress? Asian J Androl. 2015;17:633-9.

[5] Cerolini S, Maldjian A, Pizzi F, Gliozzi TM. Changes in sperm quality and lipid composition during cryopreservation of boar semen. Reproduction. 2001;121:395-401.

[6] Brouwers JF, Silva PF, Gadella BM. New assays for detection and localization of endogenous lipid peroxidation products in living boar sperm after BTS dilution or after freeze-thawing. Theriogenology. 2005;63:458-69.

[7] Saleh RA, Agarwal A. Oxidative stress and male infertility: from research bench to clinical practice. J Androl. 2002;23:737-52.

[8] Aitken RJ, Gibb Z, Baker MA, Drevet J, Gharagozloo P. Causes and consequences of oxidative stress in spermatozoa. Reprod Fertil Dev. 2016;28:1-10.

[9] Bathgate R. Antioxidant mechanisms and their benefit on post-thaw boar sperm quality. Reprod Domest Anim. 2011;46 Suppl 2:23-5.

[10] Mann, T and Lutwak-Mann, C. Male Reproductive Function and Semen. 1st ed. New York: Springer-Verlag Berlin Heidelberg; 1981.

[11] Barranco I, Tvarijonaviciute A, Perez-Patiño C, Parrilla I, Ceron JJ, Martinez EA, et al. High total antioxidant capacity of the porcine seminal plasma (SP-TAC) relates to sperm survival and fertility. Sci Rep. 2015;5:18538. 
[12] García EM, Calvete JJ, Sanz L, Roca J, Martínez EA, Vázquez JM. Distinct effects of boar seminal plasma fractions exhibiting different protein profiles on the functionality of highly diluted boar spermatozoa. Reprod Domest Anim. 2009;44:200-5.

[13] Perez-Patiño C, Barranco I, Parrilla I, Valero ML, Martinez EA, Rodriguez-Martinez $\mathrm{H}$, et al. Characterization of the porcine seminal plasma proteome comparing ejaculate portions. J Proteomics. 2016;142:15-23.

[14] Saravia F, Wallgren M, Johannisson A, Calvete JJ, Sanz L, Peña FJ, et al. Exposure to the seminal plasma of different portions of the boar ejaculate modulates the survival of spermatozoa cryopreserved in MiniFlatPacks. Theriogenology. 2009;71:662-75.

[15] Alkmin DV, Perez-Patiño C, Barranco I, Parrilla I, Vazquez JM, Martinez EA, et al. Boar sperm cryosurvival is better after exposure to seminal plasma from selected fractions than to those from entire ejaculate. Cryobiology. 2014;69:203-10.

[16] Erel O. A novel automated direct measurement method for total antioxidant capacity using a new generation, more stable ABTS radical cation. Clin Biochem. 2004;37:27785.

[17] Benzie IF, Strain JJ. The ferric reducing ability of plasma (FRAP) as a measure of "antioxidant power": the FRAP assay. Anal Biochem. 1996;239:70-6.

[18] Campos C, Guzman R, Lopez-Fernandez E, Casado A. Evaluation of the copper (II) reduction assay using bathocuproinedisulfonic acid disodium salt for the total antioxidant capacity assessment: the CUPRAC-BCS assay. Anal Biochem. 2009;392:37-44.

[19] Barranco I, Roca J, Tvarijonaviciute A, Ruber M, Vicente-Carrillo A, Atikuzzaman M, et al. Measurement of activity and concentration of paraoxonase $1(\mathrm{PON}-1)$ in seminal plasma and identification of PON-2 in the sperm of boar ejaculates. Mol Reprod Dev. 2015;82:58-65. 
[20] Koppers AJ, De Iuliis GN, Finnie JM, McLaughlin EA, Aitken RJ. Significance of mitochondrial reactive oxygen species in the generation of oxidative stress in spermatozoa. J Clin Endocrinol Metab. 2008;93:3199-207.

[21] Guthrie HD, Welch GR. Determination of intracellular reactive oxygen species and high mitochondrial membrane potential in Percoll-treated viable boar sperm using fluorescence-activated flow cytometry. J Anim Sci. 2006;84:2089-100.

[22] Alkmin DV, Martinez-Alborcia MJ, Parrilla I, Vazquez JM, Martinez EA, Roca J. The nuclear DNA longevity in cryopreserved boar spermatozoa assessed using the Sperm-Sus-Halomax. Theriogenology. 2013;79:1294-300.

[23] Petrie, A., Watson P. Statistics for Veterinary and Animal Science. 3rd ed. WileyBlackwell; 2013, p. 391.

[24] Peña FJ, Johannisson A, Wallgren M, Rodriguez Martinez H. Antioxidant supplementation in vitro improves boar sperm motility and mitochondrial membrane potential after cryopreservation of different fractions of the ejaculate. Anim Reprod Sci. 2003;78:85-98.

[25] Saravia F, Wallgren M, Rodríguez-Martínez H. Freezing of boar semen can be simplified by handling a specific portion of the ejaculate with a shorter procedure and MiniFlatPack packaging. Anim Reprod Sci. 2010;117:279-87.

[26] Rodríguez-Martínez H, Kvist U, Saravia F, Wallgren M, Johannisson A, Sanz L, et al. The physiological roles of the boar ejaculate. Soc Reprod Fertil Suppl. 2009;66:1-21. [27] Roca J, Parrilla I, Bolarin A, Martinez EA, Rodriguez-Martinez H. Will AI in pigs become more efficient? Theriogenology. 2016;86:187-93.

[28] Watson PF. The causes of reduced fertility with cryopreserved semen. Anim Reprod Sci. 2000;60:481-92. 
[29] Kim S, Lee Y-J, Kim Y-J. Changes in sperm membrane and ROS following cryopreservation of liquid boar semen stored at $15^{\circ} \mathrm{C}$. Anim Reprod Sci. 2011;124:11824.

[30] Lavranos G, Balla M, Tzortzopoulou A, Syriou V, Angelopoulou R. Investigating ROS sources in male infertility: A common end for numerous pathways. Reprod. Toxicol.. 2012;34:298-307.

[31] Gosalvez J, Tvrda E, Agarwal A. Free radical and superoxide reactivity detection in semen quality assessment: past, present, and future. J Assist Reprod Genet. 2017;34:697707.

[32] Paoli D, Lombardo F, Lenzi A, Gandini L. Sperm cryopreservation: effects on chromatin structure. Adv Exp Med Biol. 2014;791:137-50.

[33] Evenson DP, Thompson L, Jost L. Flow cytometric evaluation of boar semen by the sperm chromatin structure assay as related to cryopreservation and fertility. Theriogenology. 1994;41:637-51.

[34] Hernández M, Roca J, Ballester J, Vázquez JM, Martínez EA, Johannisson A, et al. Differences in SCSA outcome among boars with different sperm freezability. Int $\mathrm{J}$ Androl. 2006;29:583-91.

[35] Gosalvez J, Nunez R, Fernandez JL, Lopez-Fernandez C, Caballero P. Dynamics of sperm DNA damage in fresh versus frozen-thawed and gradient processed ejaculates in human donors. Andrologia. 2011;43:373-7.

[36] Koziorowka-Gilun M, Koziorowski M, Strzezek J, Fraser L. Seasonal changes in antioxidant defence systems in seminal plasma and fluids of the boar reproductive tract. Reprod Biol. 2011;11:37-47. 
[37] Vilagran I, Yeste M, Sancho S, Castillo J, Oliva R, Bonet S. Comparative analysis of boar seminal plasma proteome from different freezability ejaculates and identification of Fibronectin 1 as sperm freezability marker. Andrology. 2015;3:345-56.

[38] Wysocki P, Orzołek A, Strzeżek J, Koziorowska-Gilun M, Zasiadczyk Ł, Kordan W. The activity of $\mathrm{N}$-acetyl- $\beta$-hexosaminidase in boar seminal plasma is linked with semen quality and its suitability for cryopreservation. Theriogenology. 2015;83:1194202.

[39] Corcini CD, Varela AS, Pigozzo R, Rambo G, Goularte KL, Calderam K, et al. Prefreezing and post-thawing quality of boar sperm for distinct portions of the ejaculate and as a function of protein bands present in seminal plasma. Livest Sci. 2012;145:28-33.

[40] Fraga CG, Oteiza PI, Galleano M. In vitro measurements and interpretation of total antioxidant capacity. Biochim Biophys Acta. 2014;1840:931-4.

[41] Roca J, Rodriguez MJ, Gil MA, Carvajal G, Garcia EM, Cuello C, et al. Survival and in vitro fertility of boar spermatozoa frozen in the presence of superoxide dismutase and/or catalase. J Androl. 2005;26:15-24.

[42] Waheed MM, Gouda EM, Khalifa TA. Impact of seminal plasma superoxide dismutase and glutathione peroxidase on cryopreserved buffalo spermatozoa. Anim Reprod Sci. 2013;142:126-30.

[43] Kowalowka M, Wysocki P, Fraser L, Strzezek J. Extracellular superoxide dismutase of boar seminal plasma. Reprod Domest Anim. 2008;43:490-6.

[44] Agarwal A, Virk G, Ong C, du Plessis SS. Effect of oxidative stress on male reproduction. World J Mens Health. 2014;32:1-17.

[45] Barranco I, Tvarijonaviciute A, Perez-Patiño C, Alkmin DV, Ceron JJ, Martinez EA, et al. The activity of paraoxonase type $1(\mathrm{PON}-1)$ in boar seminal plasma and its 
relationship with sperm quality, functionality, and in vivo fertility. Andrology. 2015;3:315-20.

[46] Brigelius-Flohe R, Maiorino M. Glutathione peroxidases. Biochim Biophys Acta. 2013;1830:3289-303.

[47] Re R, Pellegrini N, Proteggente A, Pannala A, Yang M, Rice-Evans C. Antioxidant activity applying an improved ABTS radical cation decolorization assay. Free Radic Biol Med. 1999;26:1231-7.

[48] Niki E. Assessment of antioxidant capacity of natural products. Curr Pharm Biotechnol. 2010;11:801-9.

[49] Gadea J. Sperm factors related to in vitro and in vivo porcine fertility. Theriogenology. 2005;63:431-44.

[50] Giaretta E, Estrada E, Bucci D, Spinaci M, Rodríguez-Gil JE, Yeste M. Combining reduced glutathione and ascorbic acid has supplementary beneficial effects on boar sperm cryotolerance. Theriogenology. 2015;83:399-407.

[51] Eriksson BM, Vazquez JM, Martinez EA, Roca J, Lucas X, Rodriguez-Martinez H. Effects of holding time during cooling and of type of package on plasma membrane integrity, motility and in vitro oocyte penetration ability of frozen-thawed boar spermatozoa. Theriogenology. 2001;55:1593-605. 


\section{Figure Legends}

Figure 1. Box-whisker plots showing the generation of reactive oxygen species (ROS) and the incidence of lipid peroxidation (LPO) in frozen-thawed (FT) boar sperm from the first $10 \mathrm{ml}$ of sperm-rich ejaculate fraction (10mL-SRF), the rest of the SRF, the postSRF and the recomposed entire ejaculate (EE). Boxes enclose the 25th and 75th percentiles, the line denotes the median value, and the whiskers extend to the 5th and 95th percentiles. a-c and m-o indicate differences among semen sources at $\mathrm{P}<0.05$ and $\mathrm{P}<0.01$, respectively. Data of 10 ejaculates from 5 boars, 2 ejaculates per boar. 
Figure 2. Box-whisker plot showing the incidence of nuclear DNA fragmentation in frozen-thawed (FT) boar sperm from the first $10 \mathrm{ml}$ of sperm-rich ejaculate fraction (10mL-SRF), the rest of the SRF, the post-SRF and the recomposed entire ejaculate (EE). Boxes enclose the 25th and 75th percentiles, the line shows the median, and the whiskers extend to the 5th and 95th percentiles. Data of 10 ejaculates from 5 boars, 2 ejaculates per boar.

Figure 3. Box-whisker plots showing the total antioxidant capacity of boar seminal plasma from the first $10 \mathrm{~mL}$ of the sperm-rich ejaculate fraction (10 mL-SRF), the rest of the SRF, the post-SRF and the recomposed entire ejaculate (EE). Total antioxidant capacity was measured in terms of trolox equivalent antioxidant capacity (TEAC), ferric reducing ability of plasma (FRAP) and cupric reducing antioxidant capacity (CUPRAC). Boxes enclose the 25th and 75th percentiles, the line shows the median value, and the whiskers extend to the 5th and 95th percentiles. a,b; m,n and $\mathrm{x}-\mathrm{z}$ indicate differences among semen sources at $\mathrm{P}<0.05, \mathrm{P}<0.01$ and $\mathrm{P}<0.001$, respectively. Data of 10 ejaculates from 5 boars, 2 ejaculates per boar.

Figure 4. Box-whisker plots showing the activity of superoxide dismutase, catalase, paraoxonase type 1 and glutathione peroxidase and the concentration of glutathione peroxidase in 5 in boar seminal plasma from the first $10 \mathrm{~mL}$ of sperm-rich ejaculate fraction (10 mL-SRF), the rest of the SRF, the post-SRF and the recomposed entire ejaculate (EE). Boxes enclose the 25th and 75th percentiles. The line shows the median value, while the whiskers extend to the 5th and 95th percentiles. a, b and m-p indicate 
differences among semen sources at $\mathrm{P}<0.05$ and $\mathrm{P}<0.01$, respectively. Data of 10 ejaculates from 5 boars, 2 ejaculates per boar. 